\title{
EVOLUTION OF DIFFERENT BACTERIAL MNINGOENGEPHALITIS: SERIES OF CASE PRESENTATIONS AND LITERATURE REVIEW
}

\section{Cambrea Simona Claudiai,2, Marcas Consuela2, Diaconu Simona2, Mihai Raluca2, Pinzaru Anca Daniela3,4, Mangu Florin ${ }^{3,4}$, Mihai Larisia4}

${ }^{1}$ Infectious Diseases Clinic, Faculty of Medicine, "Ovidius" University, Constanta, Romania

${ }^{2}$ Clinical Infectious Diseases Hospital of Constanța, Romania

${ }^{3}$ Clinic of Pediatrics, Faculty of Medicine, "Ovidius" University, Constanta, Romania

${ }^{4}$ Emergency County Clinical Hospital Constanta “Sf. Ap. Andrei”, Constanta, Romania

\begin{abstract}
$\overline{\text { ABSTRACT }}$
Bacterial meningitis is one of the most important medical emergencies, a life-threatening condition that leads to death in all the cases in untreated patients. In infants and young children, especially under 5 years old, the most encountered and severe forms are caused by Streptococcus pneumoniae, Neisseria meningitidis and Hemophilus influenzae type $b$. The risk of neurological impairment after an acute episode of bacterial meningitis is relatively high. Worldwide, prevention through vaccination decreased dramatically incidence and mortality related to these disease. In Romania vaccination for Neisseria meningitides is optional available for just 6 months, vaccination for Streptococcus pneumoniae by one year, and for Haemophylus influenzae type $B$ is available over 10 years.

We present evolution of a series of three cases with different bacterial meningoencephalitis with severe evolution and prolonged hospitalization. All these cases were reported in unvaccinated children for germs that cause meningitis.
\end{abstract}

Keywords: bacterial meningitis, vaccination, children, neurologic complications, seizures

\section{Introduction}

Worldwide, prevention of different type of infectious diseases through vaccination lead to disappearance of polio, measles, rubella, or diphtheria. The most important result, saving millions of lives. Unfortunately, in the last decade a new trend is developing: anti-vaccination movement. Based on misinformed, ignorant and misinterpreted hypotheses the anti-vaccination activists led to large masses of unvaccinated children, therefor-eradicated diseases started to reappear $(1,2)$.

In the following article we emphasis the effect of preventable diseases that led to important health consequences.

Bacterial meningitis is considered one of the most important medical emergencies. In infants and young children, especially under 5 years old, the most encountered and severe forms are determined by Streptococcus pneumoniae, Neisseria meningitidis and Hemophilus influenzae type b (2-6). A life-threatening condition, bacterial meningitis, leads to death in all the cases if the patient does not receive appropriate antibiotic treatment. The risk of neurological impairment after an acute episode is reported at $20 \%$, worldwide $(7,8,9)$.

In Romania, vaccination for Haemophylus influenza type B is available for a few years and 
for Streptococcus pneumoniae for over one year vaccination but for Neisseria meningitides is available for just 6 months, and not included in the national program of immunization.

The series of the next three case presentation were published with parents inform consent taken in the moment of hospitalization.

\section{Case report 1}

A 9- year- old boy is presented to hospital for: headache, fever, left convergent strabismus, diplopia and left facial paralysis. The child presented 10 days before hospitalization signs of otitis and received oral antibiotics for 5 days. The clinical evolution was progressively aggravating - vomiting, intensive headache, walking instability, diplopia, facial paralysis. The medical history did not reveal other chronic diseases. At the physical examination, the patient presented pallor, sleepiness, bradylalia, tachypnea, signs of dehydration. The neurological exam revealed decreased level of consciousness, left facial paralysis, left convergent strabismus, positive signs of meningeal irritation (neck stiffness).

The cell counts underlined leukocytosis with neutrophilia $(16.63 \times 109 / \mathrm{L}$ comparative with normal range 5-14.5×109/L), anemia, thrombocytosis and high inflammatory markers: erythrocyte sedimentation rate (ESR) $115 \mathrm{~mm} / 1$ hour (normal range $9-10 \mathrm{~mm} / 1$ hour), $\mathrm{C}$ reactive protein (CRP) $285 \mathrm{mg} / \mathrm{l}$ (normal range $0-5 \mathrm{mg} / \mathrm{l}$ ), fibrinogen $663 \mathrm{mg} / \mathrm{dl}$ (normal value 150-400 mg/ dl. Blood urea, serum creatinine, liver enzymes, electrolytes were within normal range.

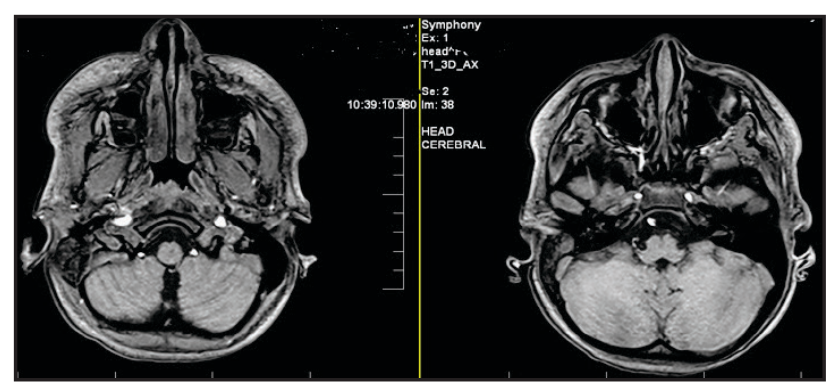

Figure 1 Cerebral IRM-right mastoiditis; the other cerebral structures are normal.

Thelumbarpuncture performed shortly after admission presented opalescent cerebrospinal fluid (CSF), pleocytosis (4225 cells/mmc, more polymorphonuclear, comparative with normal range: 0-5 cells $/ \mathrm{mmc}$ ). Biochemical analysis of CSF revealed high levels of proteins (1506 $\mathrm{mg} / \mathrm{L}$, normal value: $80-320 \mathrm{mg} / \mathrm{L}$ ) and low level of glucose. CSF culture isolated Streptococcus pneumoniae.

The diagnosis is meningoencephalitis and sepsis with Streptococcus pneumoniae due to untreated ear infection, in a child without vaccination for Streptococcus pneumoniae.

Antibiotic therapy with Meropenem, Vancomycin and Amikacin was administered since the first hours of hospitalization, corticosteroids, drugs that reduce cerebral edema, symptomatic and supportive treatment. After 7 days of treatment, the patient presents generalized convulsions as consequence of cerebral edema (confirmed by an MRI evaluation), hypokalemia. The seizures were associated with high blood pressure $(180 / 120 \mathrm{mmHg})$ and bradycardia. The patient was transferred to the Intensive Care Unit for permanent and careful surveillance. At the previous treatment was added, at the recommendation of pediatric neurologist, antiepileptic medication.

After this point the evolution was favorable, with apparently complete recovery, but with changes evidenced by EEG (slow theta wave and rare spike and wave discharge in left temporal area). Anti-epileptic treatment that was initiated during hospitalisation was recommended for at least next 3 months. Although the child should be evaluated after one month of discharge, from neurologic point of view he was lost from our evidence.

\section{Case report 2}

An immunocompetent 5-year-old boy is presented in the emergency room for: high fever, rash, headache, vomiting and photophobia. The patient presented altered general condition, fever, vomiting and after 12 hours the rash appeared. The cutaneous maculo-papular, and purpuric rash extended rapidly, covering all 4 limbs and the torso (Figure 2). 


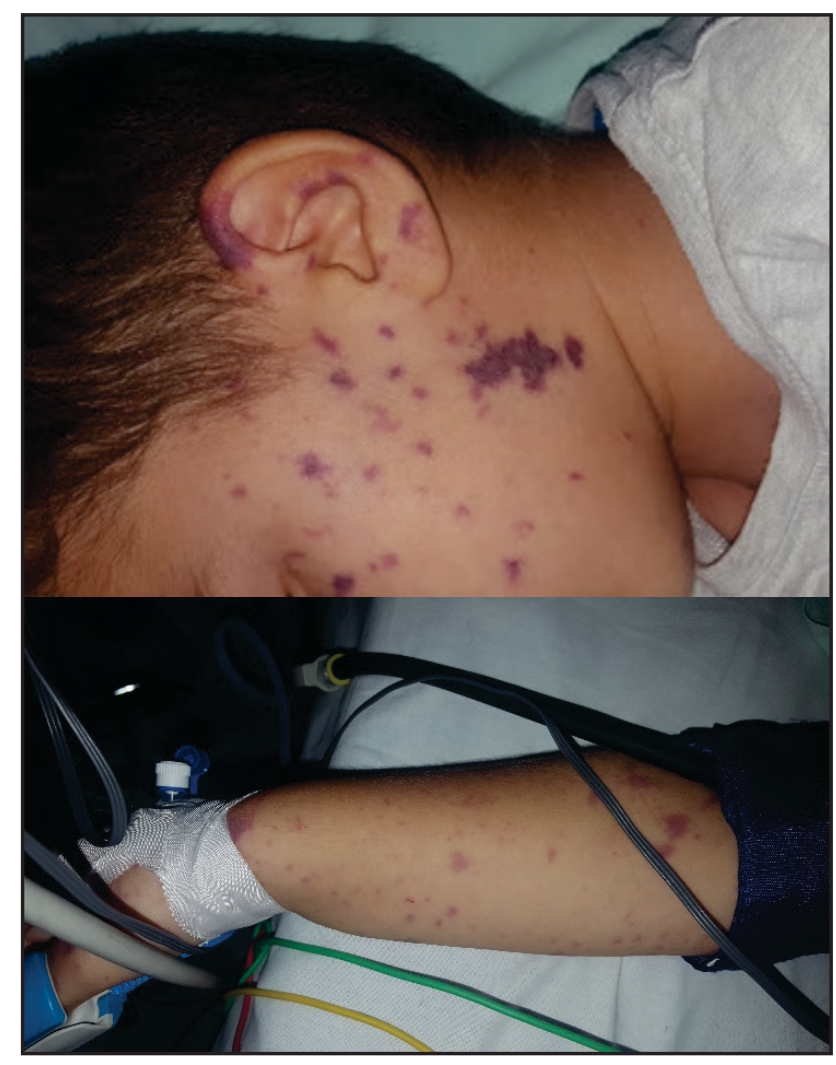

Figure 2 Maculo-papular and purpuric rash

At admission the general state was altered, hypotonia, muscle pain in upper and lower limbs, tachypnea, fine crackles at lung auscultation, frequent cough, diffuse abdominal pain, photophobia, headache and neck rigidity.

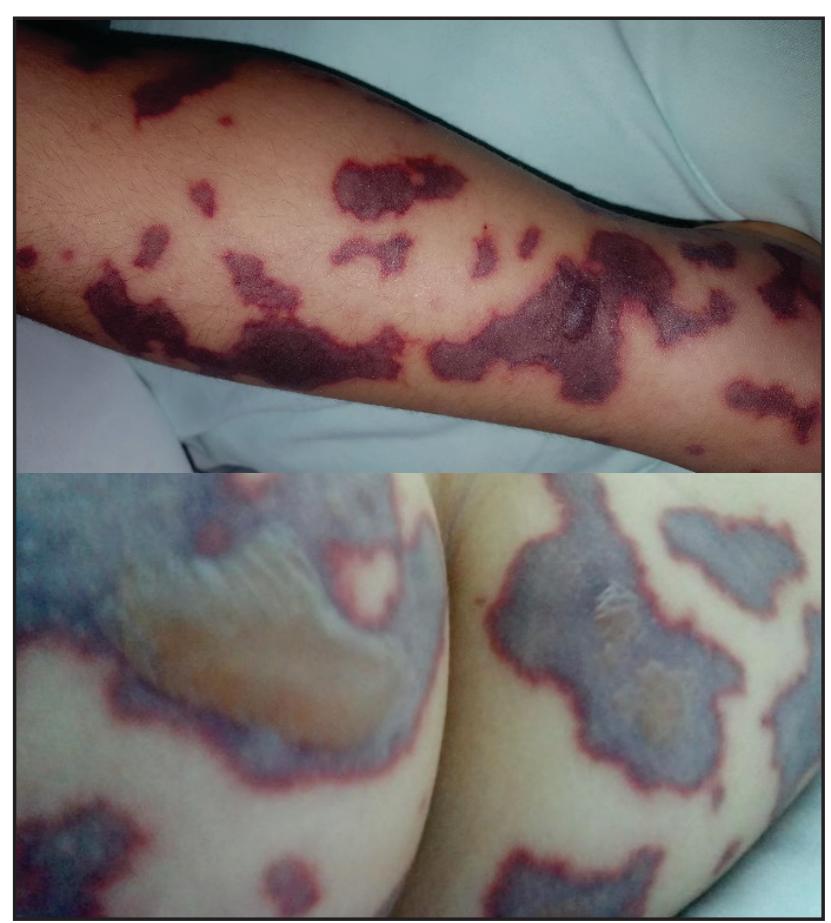

Figure 3 Cutaneous Blisters

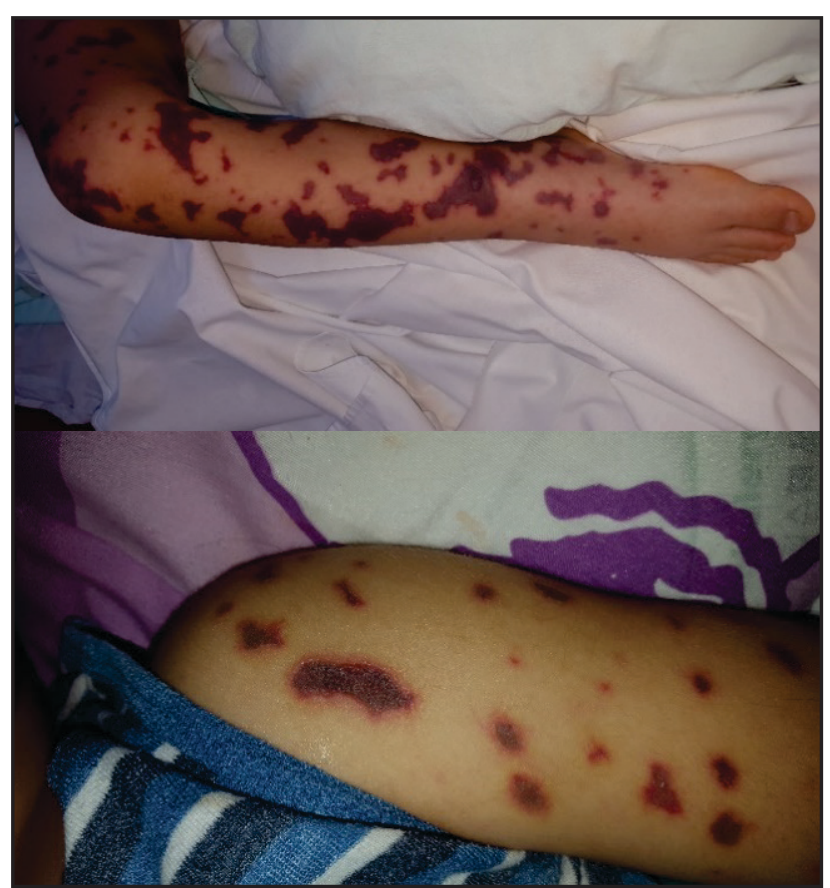

Figure 4 Ecchymotic lesions in evolution

The cell counts revealed leukocytosis $(32.62 \times 109 / \mathrm{L}$; normal range 5.5-15.5×109/L) with increased level of neutrophils, anemia, thrombocytopenia $(57.000 / \mathrm{mmc}$, comparative with normal range $150000-400000 / \mathrm{mmc}$ ) and high inflammatory markers: $E S R=20 \mathrm{~mm} / 1$ $\mathrm{h}, \mathrm{CRP}=102 \mathrm{mg} / \mathrm{l}$, fibrinogen $1369 \mathrm{mg} / \mathrm{dl}$. CSF culture and nasal culture was positive for Neisseria meningitidis.

The diagnosis was meningococcemia with fulminans purpura. The patient was isolated in a separate room and antibiotic therapy is initiate with Meropenem, and Vancomycin. Also we administered antifungal (Fluconazole), diuretics (Furosemide, Mannitol), immunoglobulin and plasma. After 5 days, the cutaneous lesions became blisters, as we can see in Figure 3. The evolution of cutaneous lesions was slowly favorable as it is evidenced in Figure 4, after 18 days of evolution. The patient was discharged after 35 days of treatment, without neurologic consequences on short term, but with important cutaneous scars.

\section{Case report 3}

A 4-year-old boy, from rural area, unvaccinated, is presented to our department for fever and headache. 24 hours prior to admission the symptoms aggravated therefore he was oriented to the emergency room. The examination revealed: altered general condition, intermittent 
agitation, headache and neck rigidity, positive Kerning sign, photophobia, anisocoria, left periorbital edema, coffee ground vomitus.

The laboratory analysis revealed leukocytosis $(17.2 \times 109 / \mathrm{L}$ comparative with normal value $5.5-15.5 \times 109 / \mathrm{L})$ with increased neutrophils value, anemia, thrombocytopenia $(86.000 / \mathrm{mmc})$ and high inflammatory markers: ESR $130 \mathrm{~mm} / \mathrm{l} \mathrm{h}$, CRP $6 \mathrm{mg} / \mathrm{l}$, and fibrinogen $1595 \mathrm{mg} / \mathrm{dl}$. Moderate dehydration with slightly elevated urea. The lumbar puncture evidenced opalescent CSF, pleocytosis (14270 cells/mm3, with predominance of polymorphonuclear leucocytes). Biochemical analysis revealed high levels of proteins $(1320 \mathrm{mg} / \mathrm{dl})$ and low level of glucose. CSF culture was positive for Hemophilus influenzae.

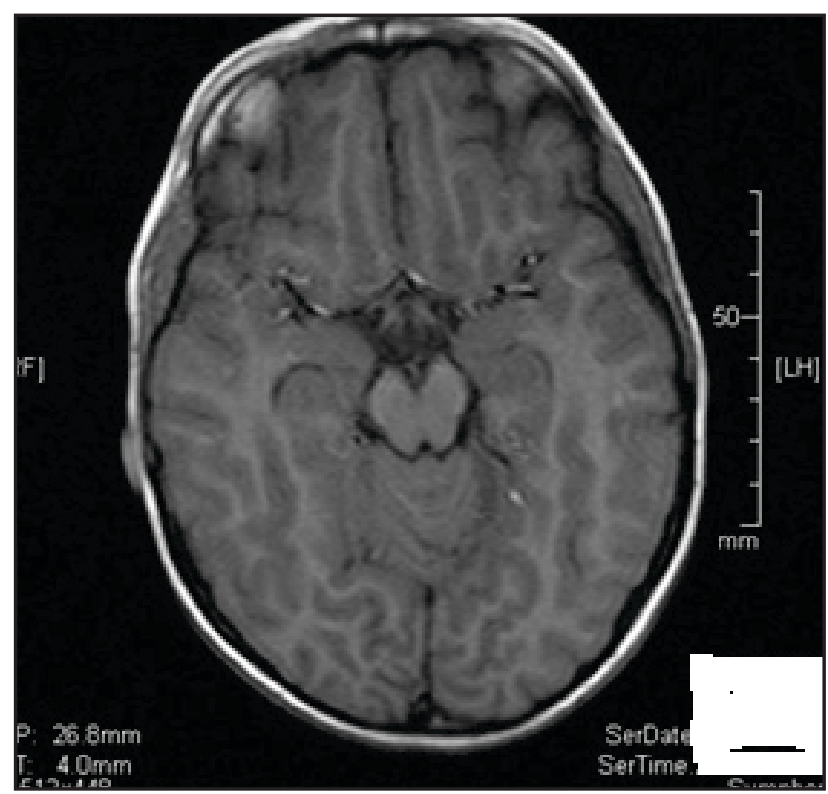

Figure 5 Cerebral MRI-subdural fronto-temporo-parietal bilateral effusion; the other cerebral structures are normal.

The diagnosis of meningitidis with Hemophilus influenzae was established. After isolation, the antibiotic therapy is initiated (Meropenem, and then Ampicillin with Gentamycin) in association with corticosteroids, diuretics, and drugs that decreased cerebral edema, electrolytes and gastric protection. The cerebral magnetic resonance imaging (MRI) evidenced a subdural fronto-temporo-parietal bilateral effusion for which was taken into consideration a neurosurgical drainage. After 45 days of antibiotic and symptomatic treatment the subdural effusion regressed, and the patient was discharged without any sequels.

\section{Discussion}

Mandatory immunization of the children determined an important change of the incriminated agents that could produce meningitidis. Initial Hemophilus type $b$ was the most encountered, but after vaccination became universal its place was occupied be streptococcus pneumoniae. Isaacman et al., analyzed retrospectively the importance of 7-valent pneumococcal conjugate vaccine in Europe. After reporting an annual incidence of 44.4/100000 invasive pneumococcal diseases for almost 20 years after introducing mandatory vaccination the percentage dropped dramatically. Black et al., confirmed a more than $90 \%$ reduction. Introducing 13-valent pneumococcal conjugate vaccine demonstrated a greater decrease. Cohan et al analyzed 963 swabs form children with ages between 6-24 months, 285 receiving 7 -valent and the rest 13-valent pneumococcal conjugate vaccine. The result demonstrated that invasive pneumococcal diseases were extremely lower in the first group. Therefore, 13-valent pneumococcal conjugate vaccine could assure a more efficient coverage (10-14)

The clinical presentation of meningitis is variable with age. Smaller children will present more atypical elements; therefore, the diagnosis could be delayed. The most important step in preventing or limiting the neurological impact of bacterial meningitis is establishing rapidly and correctly the antibiotic therapy (10-14).

Certain diseases amplify the risk of developing bacterial meningitis, such as: otitis or recurrent otitis, sinusitis, mastoiditis, congenital malformations, any shunt that involves the CSF drainage, invasive head therapy (surgery, or trauma) (10-14).

Ceri et al., demonstrated that inflammatory processes of the brain that evolves with seizure is a sign of negative prognosis. In our case the patient presented after 7 days of treatment seizures. At the end of therapy, the patient presented EEG abnormalities that imposed treatment and a quarter follow-up for one year (15).

CSF high protein levels could be considered 
an important predictable factor for cerebral parenchyma damage. Kim et al., underlined this element. Also, Tan et al., analyzed 232 neonates with bacterial meningitidis suggesting that high level of protein in CSF were encountered in patients that developed neurological manifestations with long term sequels (mental retardation, motor disorders). There are yet ambiguities regarding this subject (16).

Our first patient, hospitalized with Pneumococcal meningitis presented a high level of proteins in CSF $(1506 \mathrm{mg} / \mathrm{dl})$ in association with another negative prognostic factor seizure. This child did not received any vaccine for pneumococcal invasive disease. In this case we could not monitor the possible neurological sequelae because the patient did not return to control in our clinic.

Neisseria meningitidis is a gram-negative bacterium with 12 serogroups, from which only six are responsible for the severest forms of meningitis (A, B, C, W, X, Y). In Romania is available only one vaccine for serogroups: A, C, $\mathrm{Y}$, and W135. It is not yet included in the national vaccination program. For B serotype, the vaccine is not available in our country, but in European Community it is mandatory. Maiden et al analyzed 43.309 patient under 19-year-old, from which 8599 were positive for meningococci. All received anti-meningococcal $\mathrm{C}$ vaccine. The carriage became lower by $81 \%$. The death rate decreased dramatically (one case in 2009) $(17,18)$.

Our second case, presented in this paper, was a child from collectivity - kindergarten who did not receive a vaccine for meningococcal disease before this episode of meningococcemia. Although the child evolution for neurological point of view was favorable, the cutaneous lesion presented slow evolution towards healing.

Brouwer et al published in 2015 a more elaborate Cochrane metanalysis regarding the benefit of corticosteroids therapy in bacterial meningitidis. After comparing 25 studies (2511 children) it underlined that mortality rate was not diminished, but neurological sequels were reduced (19).

Hemophilus influenzae represents one of the most aggressive bacterial infections encountered in pediatrics, responsible for many bacterial meningitidis and pneumonia in small children. The sequels induced by the Hemophilus infection are mainly neurological (starting with paralysis, mental retardation, deafness, psychological disabilities) (20-26).

Introduce in the early 1990s it reduced dramatically the incidence of Hemophilus infections. Cowgill et col., analyzed the immunization response in Kenya reveling 88\% effectiveness for children under 5-year-old and $87 \%$ for children under 2-year-old. Similar results were noticed in USA $(92 \%)$ and UK $(87 \%)$. One of the most important examples is offered by Gambia; in this country between 1997 and 2002 vaccination for Hemophilus was introduced in general plan of immunization. After 2002 the annual incidence of meningitidis due to Hemophilus dropped to zero for children under 5-year-old (27-32).

In the last 10 years in our area this child was the only one with meningitis with Hemophilus influenzae. The disease was noticed in a child who did not receive all vaccination, nor the one for Hemophylus. The evolution was slowly favorable under conservative treatment although it presented subdural empyema.

\section{Conclusions}

The presented cases should be considered as an alarming sign. The neurological impact could be severe, in all bacterial meningitis cases and the long-term sequels could be permanent and life threating.

In order to prevent longer hospitalization and complex medical approach of children with bacterial meningitis, immunization in childhood for prevention of these severe infections is an important alternative. Meningococcal vaccination should be included in our national program of immunization as soon as possible like haemophilus and pneumococcal vaccination.

\section{References}

1. Centers for disease Control and Prevention. Why Are Childhood Vaccines So Important? 2018 [cited 2019]; Available from: https:// www.cdc.gov/vaccines/vac-gen/howvpd. htm. 
2. Pick AM, Sweet DC, Begley KJ. A review of pediatric bacterial meningitis. US Pharmacist. 2016;41(5):41-5.

3. Theodoridou MN, Vasilopoulou VA, Atsali EE, Pangalis AM, Mostrou GJ, Syriopoulou VP, et al. Meningitis registry of hospitalized cases in children: epidemiological patterns of acute bacterial meningitis throughout a 32-year period. BMC Infect Dis. 2007 Aug 30;7:101.

4. Agrawal S, Nadel S. Acute bacterial meningitis in infants and children: epidemiology and management. Paediatr Drugs. 2011 Dec 1;13(6):385-400.

5. de Jonge RC, van Furth AM, Wassenaar M, Gemke RJ, Terwee CB. Predicting sequelae and death after bacterial meningitis in childhood: a systematic review of prognostic studies. BMC Infect Dis. 2010 Aug 5;10:232.

6. Sheldon L, Kaplan MD. Bacterial meningitis in children older than one month: Clinical features and diagnosis. UpToDate [cited 2019]; Available from: https://www. uptodate.com/contents/bacterial-meningitisin-children-older-than-one-month-clinicalfeatures-and-diagnosis.

7. Tacon CL, Flower O. Diagnosis and management of bacterial meningitis in the paediatric population: a review. Emerg Med Int. 2012;2012:320309.

8. Edmond K, Clark A, Korczak VS, Sanderson C, Griffiths UK, Rudan I. Global and regional risk of disabling sequelae from bacterial meningitis: a systematic review and meta-analysis. Lancet Infect Dis. 2010 May;10(5):317-28.

9. Kulkarni G. A case report of acute pediatric bacterial meningitis due to the rare isolate, Pseudomonas putida. Neuroimmunology and Neuroinflammation. 2016;3:215-8.

10. Isaacman DJ, McIntosh ED, Reinert RR. Burden of invasive pneumococcal disease and serotype distribution among Streptococcus pneumoniae isolates in young children in Europe: impact of the 7-valent pneumococcal conjugate vaccine and considerations for future conjugate vaccines. Int J Infect Dis. 2010 Mar;14(3):e197-209.

11. Kaman A, Aydin-Teke T, Oz FN, Bayhan GI, Metin O, Gayretli-Aydin ZG, et al. A pediatric case of acute meningitis due to Streptococcus pneumoniae serotype 33D. Turk J Pediatr. 2015 May-Jun;57(3):304-7.

12. Black S, Shinefield H, Fireman B, Lewis E, Ray P, Hansen JR, et al. Efficacy, safety and immunogenicity of heptavalent pneumococcal conjugate vaccine in children. Northern California Kaiser Permanente Vaccine Study Center Group. Pediatr Infect Dis J. 2000 Mar;19(3):187-95.

13. Esposito S, Principi N. Impacts of the 13-Valent Pneumococcal Conjugate Vaccine in Children. J Immunol Res. 2015;2015:591580.

14. Levy C, Varon E, Picard C, et al. Trends of pneumococcal meningitis in children after introduction of the 13-valent pneumococcal conjugate vaccine in France. Pediatr Infect Dis J. 2014; 33(12): 1216-21.

15. Cerri C, Caleo M, Bozzi Y. Chemokines as new inflammatory players in the pathogenesis of epilepsy. Epilepsy Res. 2017 Oct;136:7783.

16. Kim KS. Acute bacterial meningitis in infants and children. Lancet Infect Dis. 2010 Jan;10(1):32-42.

17. Maiden MC, Ibarz-Pavon AB, Urwin R, Gray $\mathrm{SJ}$, Andrews NJ, Clarke SC, et al. Impact of meningococcal serogroup $\mathrm{C}$ conjugate vaccines on carriage and herd immunity. J Infect Dis. 2008 Mar 1;197(5):737-43.

18. Sigurdardottir ST, Davidsdottir K, Arason VA, Jonsdottir O, Laudat F, Gruber WC, et al. Safety and immunogenicity of CRM197conjugated pneumococcal-meningococcal $\mathrm{C}$ combination vaccine (9vPnC-MnCC) whether given in two or three primary doses. Vaccine. 2008 Aug 5;26(33):4178-86.

19. Brouwer MC, McIntyre P, Prasad K, van de Beek D. Corticosteroids for acute bacterial meningitis. Cochrane Database Syst Rev. 2015 Sep 12(9):CD004405.

20. Ahmed AS, Khan NZ, Hussain M, Amin MR, Hanif M, Mahbub M, et al. Followup of cases of Haemophilus influenzae type $b$ meningitis to determine its longterm sequelae. J Pediatr. 2013 Jul;163(1 Suppl):S44-9.

21. Forgie IM, O’Neill KP, Lloyd-Evans N, Leinonen $\mathrm{M}$, Campbell $\mathrm{H}$, Whittle $\mathrm{HC}$, et 
al. Etiology of acute lower respiratory tract infections in Gambian children: I. Acute lower respiratory tract infections in infants presenting at the hospital. Pediatr Infect Dis J. 1991 Jan;10(1):33-41.

22. Forgie IM, O’Neill KP, Lloyd-Evans N, Leinonen $\mathrm{M}$, Campbell $\mathrm{H}$, Whittle $\mathrm{HC}$, et al. Etiology of acute lower respiratory tract infections in Gambian children: II. Acute lower respiratory tract infection in children ages one to nine years presenting at the hospital. Pediatr Infect Dis J. 1991 Jan;10(1):42-7.

23. Shann F, Gratten M, Germer S, Linnemann V, Hazlett D, Payne R. Aetiology of pneumonia in children in Goroka Hospital, Papua New Guinea. Lancet. 1984 Sep 8;2(8402):537-41.

24. Saha SK, Rikitomi N, Ruhulamin M, Watanabe K, Ahmed K, Biswas D, et al. The increasing burden of disease in Bangladeshi children due to Haemophilus influenzae type b meningitis. Ann Trop Paediatr. 1997 Mar;17(1):5-8.

25. Watt JP, Levine OS, Santosham M. Global reduction of Hib disease: what are the next steps? Proceedings of the meeting Scottsdale, Arizona, September 22-25, 2002. J Pediatr. 2003 Dec;143(6 Suppl):S163-87

26. Peltola H. Worldwide Haemophilus influenzae type $\mathrm{b}$ disease at the beginning of the 21st century: global analysis of the disease burden 25 years after the use of the polysaccharide vaccine and a decade after the advent of conjugates. Clin Microbiol Rev. 2000 Apr;13(2):302-17.

27. World Health Organization. Haemophilus influenzae type b (Hib) 2019 [cited 2019]; Available from: http://www.euro.who.int/en/ health-topics/disease-prevention/vaccinesand-immunization/vaccine-preventablediseases/haemophilus-influenzae-type-b-hib.

28. Cowgill KD, Ndiritu M, Nyiro J, Slack MPE, Chiphatsi S, Ismail A, et al. Effectiveness of Haemophilus influenzae type b Conjugate vaccine introduction into routine childhood immunization in Kenya. JAMA. 2006;296(6):671-8.

29. Lagos R, Horwitz I, Toro J, San Martin $\mathrm{O}$, Abrego P, Bustamante C, et al. Large scale, postlicensure, selective vaccination of Chilean infants with PRP-T conjugate vaccine: practicality and effectiveness in preventing invasive Haemophilus influenzae type b infections. Pediatr Infect Dis J. 1996 Mar;15(3):216-22.

30. Murphy TV, White KE, Pastor P, Gabriel L, Medley F, Granoff DM, et al. Declining incidence of Haemophilus influenzae type $\mathrm{b}$ disease since introduction of vaccination. Jama. 1993 Jan 13;269(2):246-8.

31. Urwin G, Yuan MF, Feldman RA. Prospective study of bacterial meningitis in North East Thames region, 1991-3, during introduction of Haemophilus influenzae vaccine. BMJ. 1994 Nov 26;309(6966):1412-4.

32. Adegbola RA, Secka O, Lahai G, LloydEvans N, Njie A, Usen S, et al. Elimination of Haemophilus influenzae type $b$ (Hib) disease from The Gambia after the introduction of routine immunisation with a Hib conjugate vaccine: a prospective study. Lancet. 2005 Jul 9-15;366(9480):144-50. 